Article

\title{
Expressing Double-Stranded RNAs of Insect Hormone-Related Genes Enhances Baculovirus Insecticidal Activity
}

\author{
Zheming Liu ${ }^{1,2,+}$, Xiaofang Wang ${ }^{1,+}$, Yan Dai ${ }^{1}$, Xiaoli Wei ${ }^{1}$, Mi Ni ${ }^{1}$, Lei Zhang ${ }^{1, *}$ and \\ Zhen Zhu ${ }^{1, *}$ \\ 1 State Key Laboratory of Plant Genomics and National Center for Plant Gene Research (Beijing), Institute of \\ Genetics and Developmental Biology, The Innovative Academy of Seed Design, Chinese Academy of \\ Sciences, Beijing 100101, China; liuzheming2011@genetics.ac.cn (Z.L.); scarlett1222@163.com (X.W.); \\ ydai@genetics.ac.cn (Y.D.); xlwei@genetics.ac.cn (X.W.); fannimi2001@gmail.com (M.N.) \\ 2 University of Chinese Academy of Sciences, Beijing 100049, China \\ * Correspondence: lzhang@genetics.ac.cn (L.Z.); zzhu@genetics.ac.cn (Z.Z.); Tel.: +86-10-6480-6616 (Z.Z.) \\ + These authors contributed equally to this work.
}

Received: 17 December 2018; Accepted: 17 January 2019; Published: 18 January 2019

\begin{abstract}
Baculoviruses have already been used for insect pest control, but the slow killing speed limits their further promotion and application. Here we provide a strategy for improving baculovirus insecticidal activity using Helicoverpa armigera nucleopolyhedrovirus (HearNPV) to express double-stranded RNAs (dsRNAs) targeting cotton bollworm (Helicoverpa armigera) juvenile hormone $(\mathrm{JH})$-related genes. Droplet-feeding bioassays show that the $50 \%$ lethal concentration $\left(\mathrm{LC}_{50}\right)$ values of recombinant baculoviruses expressing the dsRNA of $\mathrm{JH}$ acid methyl transferase gene $(H a J H A M T)$ and the JH acid binding protein gene $(H a J H B P)$ were $1.24 \times 10^{4}$ polyhedral inclusion bodies (PIB) $/ \mathrm{mL}$ and $2.26 \times 10^{4} \mathrm{PIB} / \mathrm{mL}$, respectively. Both were much lower than the control value $\left(8.12 \times 10^{4} \mathrm{PIB} / \mathrm{mL}\right)$. Meanwhile, the $\mathrm{LT}_{50}$ of recombinant baculovirus expressing dsRNA of HaJHBP was only $54.2 \%$ of the control value, which means that larval death was accelerated. Furthermore, the mRNA level of target genes was reduced in recombinant baculovirus-treated cotton bollworm larvae. Transcription of several key genes involved in hormone signaling pathways-for example, ecdysone receptor gene $(\mathrm{HaEcR})$ - was also altered. This study establishes a new strategy for pest management by interfering with insect hormone-related gene expression via baculoviruses, and the engineered baculoviruses have great potential application in cotton production.
\end{abstract}

Keywords: recombinant baculovirus; RNA interference; juvenile hormone; Helicoverpa armigera

\section{Introduction}

Insect pests cause large crop losses worldwide through direct damage and the transmission of plant diseases [1]. To date, spraying chemical insecticides remains the most common method of pest control [2], but problems such as environmental pollution, effects on human health, and the development of resistance in target pests have followed [3]. Baculoviruses, which are arthropod-specific viruses, have been successfully applied for agriculture and forestry pest management. They are safe for people and wildlife because of their extremely narrow host range [4,5]. Baculoviruses can cause epidemics in insect populations and be effective in the environment for a long time [6]. In Latin America, baculoviruses as commercial pesticides have been successfully applied in integrated pest management programs for decades in soybean and vegetable fields, as well as apple and pear orchards [7]. However, the slow killing speed (ranging from five days to more than two weeks) largely hampers their application as an insecticide $[8,9]$. Fortunately, the genome sequences of some 
notable baculoviruses were determined, and many strategies have been developed to improve their killing action and stability through recombinant DNA technology $[2,8]$. For example, recombinant baculovirus expressing an insect-specific toxin from the scorpion Androctonus australis and straw itch mite Pyemotes tritici showed improved insecticidal activity $[10,11]$.

Insect hormones play an important role in the regulation of insect growth, development, and reproduction. Insect hormone analogues, such as tebufenozide, have resulted in larval death due to the interruption of hormone-mediated cell or organ development [12]. There is considerable interest in the possibility of using insect hormones or hormone regulatory proteins in conjunction with microbial delivery systems as a means of controlling insect pests. Several hormone-related genes were used in genetically modified baculoviruses to enhance their insecticidal activity, including the genes of a diuretic hormone, eclosion hormone, prothoracicotropic hormone, and juvenile hormone $(\mathrm{JH})$ esterase [13-16]. However, just a small part of these recombinant baculoviruses showed significant improvement over parent wild-type baculoviruses in pesticidal activity. Alternatively, the deletion of a nonessential baculovirus gene coding ecdysteroid UDP-glucosyltransferase (EGT) provided a beneficial effect on killing speed [17]. EGT catalyzes the conjugation of sugar molecules to ecdysteroids, thus preventing the ecdysteroid from crossing cellular membranes [18].

RNA interference, which triggers specific gene silencing through the delivery of homologous double-stranded RNA (dsRNA) fragments [19], is widely used in biological research and shows great application potential. In agriculture, there are several successful cases of insect pest control using RNA interference (RNAi) technology [20-24]. The first RNAi-based pest control agent, a transgenic corn that produces dsRNA and two Bacillus thuringiensis (Bt) toxins against the western corn rootworm, was approved for release in the field [25]. Baculoviruses have many advantages for RNAi delivery due to their inertness, versatility, and possibility of high throughput preparation [5]. Nevertheless, research on the application of baculovirus-mediated RNAi is mainly focused on gene therapy, and there have been few reports on pest control [26].

Cotton bollworm, Helicoverpa armigera, is one of the world's most destructive pests in cotton and many other crops [27]. Helicoverpa armigera nucleopolyhedrovirus (HearNPV) has already been successfully applied to control cotton bollworms in Australia, the United States, China, and India [28]. However, since cotton bollworms do not die immediately after infection with HearNPV, it is difficult to effectively control pests and avoid losses in the outbreak period. In this work, we attempted to construct novel recombinant HearNPVs that can increase the insecticidal activity of HearNPV, and especially reduce the killing time. Our previous report showed that transgenic cotton expressing dsRNA targeting gene encoding proteins that synthesize or transport juvenile hormones was effective against cotton bollworm, especially for Bt-resistant cotton bollworm [24]. Insect resistance to the cotton-pyramiding Bt toxin and RNAi was substantially delayed compared to using Bt cotton alone. Here, to control cotton bollworm, we constructed two recombinant HearNPVs expressing dsRNA targeting JH acid methyltransferase gene (HaJHAMT) and JH-binding protein gene (HaJHBP) from $H$. armigera, and tested their insecticidal action. Our results demonstrate that the recombinant HearNPVs obtained in this study had significantly improved insecticidal activity.

\section{Results}

\subsection{Construction of Recombinant Bacmid Containing dsRNA Expression Cassette}

According to our previous report, transgenic cotton expressing dsRNA targeting H. armigera JH acid methyltransferase gene (HaJHAMT, GenBank accession number KX289532.1) or JH-binding protein gene (HaJHBP, GenBank accession number KX289533.1) showed resistance to cotton bollworm [24]. In this work, a $408 \mathrm{bp}$ fragment of HaJHAMT and a $483 \mathrm{bp}$ fragment of HaJHBP were used to compose hairpin RNAi constructs (see Supplementary Figures S1 and S2 for sequences). In order to obtain the recombinant baculovirus expressing dsRNA by the Bac-to-Bac system, the donor vectors, in which dsRNA expression was under the control of the p10 promoter and op166 
promoter (Figure 1), were constructed and transformed into E. coli DH10B harboring HaBacHZ8 $\triangle$ egt and a helper plasmid. After site-specific transposition, we selected recombinant baculovirus plasmid (bacmids) based on white-blue screening, and further confirmed them by PCR. Validated bacmids were named $\mathrm{HaBac} \Delta$ egtdsJHAMT and $\mathrm{HaBac} \Delta$ egtdsJHBP.

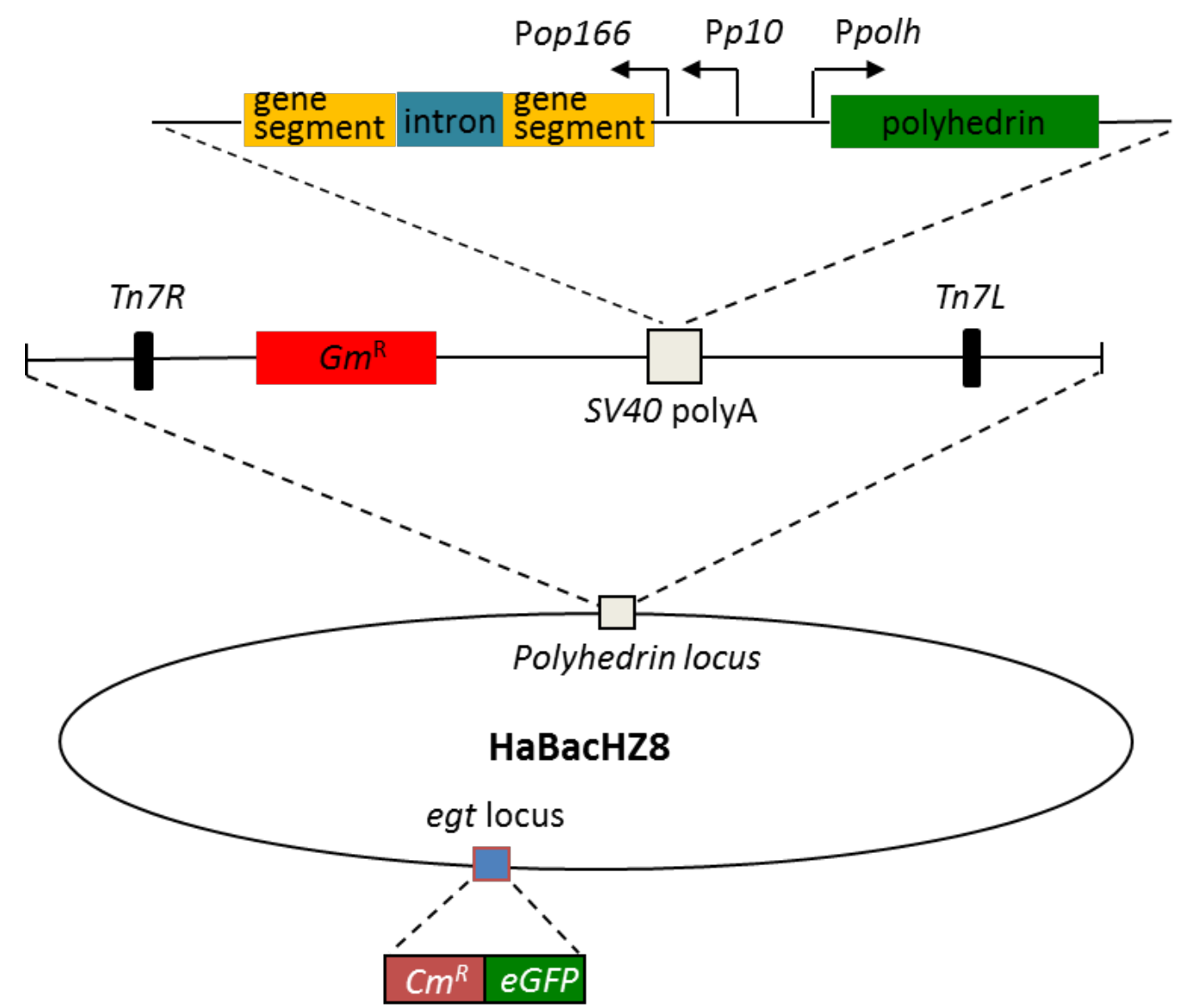

Figure 1. Schematic version of a recombinant baculovirus plasmid (bacmid). The hairpin RNA structure was composed of the same two juvenile hormone $(\mathrm{JH})$-related gene fragments in the antisense orientation separated by the intron from the potato GA20-oxidase gene. Double-stranded RNA (dsRNA) expression was controlled by the op166 and $p 10$ promoters. The polyhedrin gene under its native promoter was reinserted in recombinant bacmids. In addition, the egt locus was replaced by eGFP and $C m^{R}$ genes.

\subsection{Detection of Recombinant Baculoviruses in Transfected Cells}

To investigate the effect of the dsRNA insertion on virus infection and occlusion body (OB) formation, we transfected insect cells (HzAM1 cells), with HaBac $\Delta$ egtdsJHAMT, HaBac $\Delta$ egtdsJHBP, and $\mathrm{HaBac} \Delta$ egt (as a control). Since the egt gene locus of HaBac $\Delta$ egt was replaced by the eGFP gene, green fluorescence should be observed in the infected cells. As expected, strong green fluorescence was observed under ultraviolet light in the HzAM1 cells at three days post-transfection (Figure 2). At the same time, a typical cytopathic effect, OB formation within the cells, was also observed on bright field. 


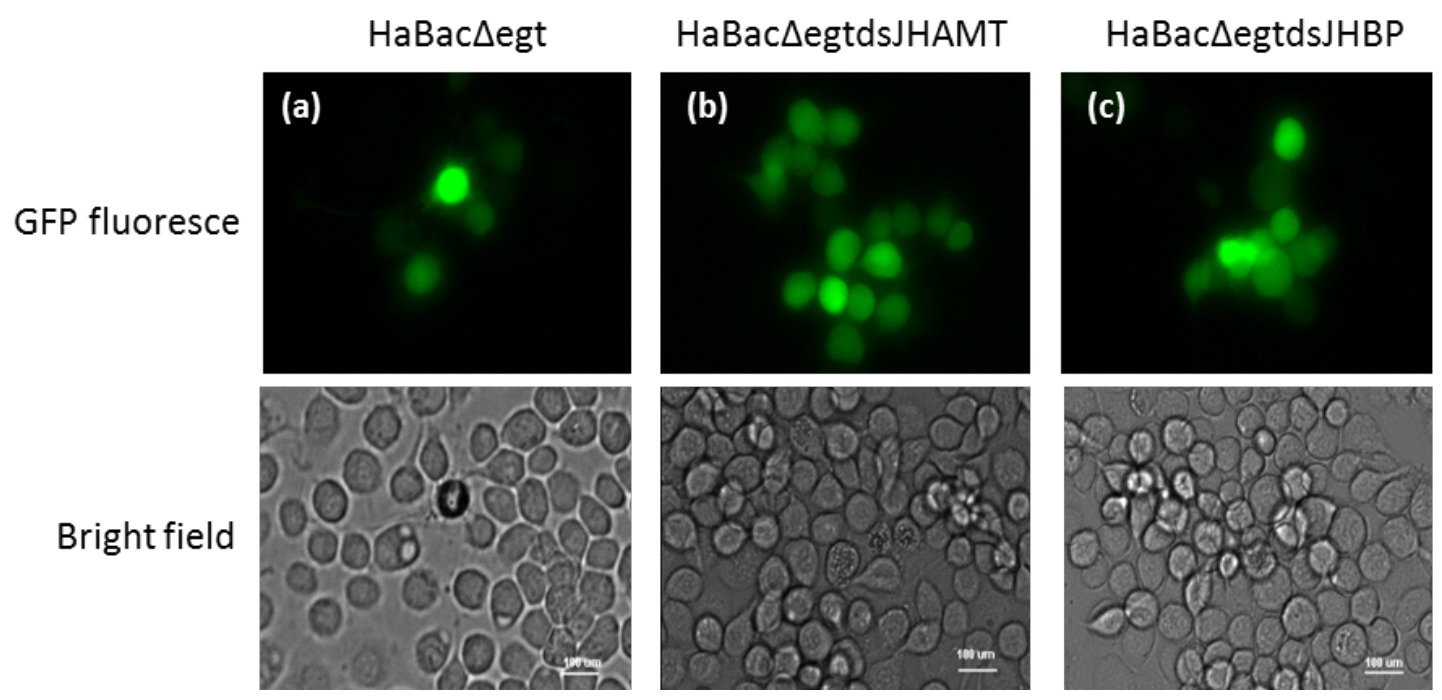

Figure 2. Microscopy observation of virus-transfected HzAM1 cells. HzAM1 cells were transfected with (a) HaBac $\Delta$ egt; (b) HaBac $\Delta$ egtdsJHAMT; or (c) HaBac $\Delta$ egtdsJHBP. Pictures were taken three days post-transfection under ultraviolet light (top panels) or visible light (bottom panels). Bars $=100 \mu \mathrm{m}$.

Furthermore, we extracted the total RNA of these infected cells and detected the transcription of dsRNA through reverse transcription PCR (RT-PCR). A 419-bp band of dsJHAMT-specific product was detectable in cells infected with HaBac $\Delta$ egtdsJHAMT (Figure 3). Similarly, a 288 bp band of dsJHBP-specific product was also detected. No signal was seen in the control (cells infected with $\mathrm{HaBac} \Delta \mathrm{egt}$ ). These results confirmed again that the recombinants were constructed correctly and dsRNA was able to transcript as expected after infecting.

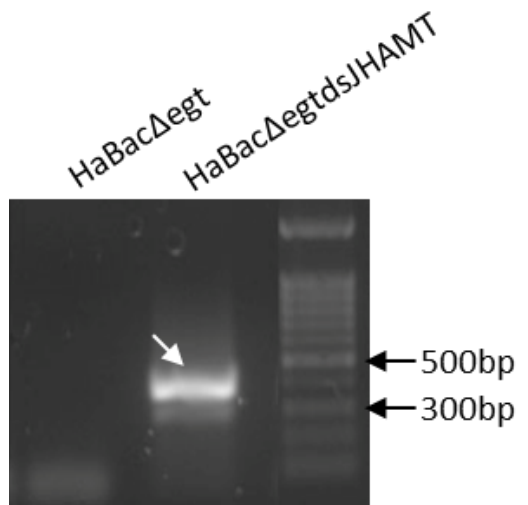

(a)

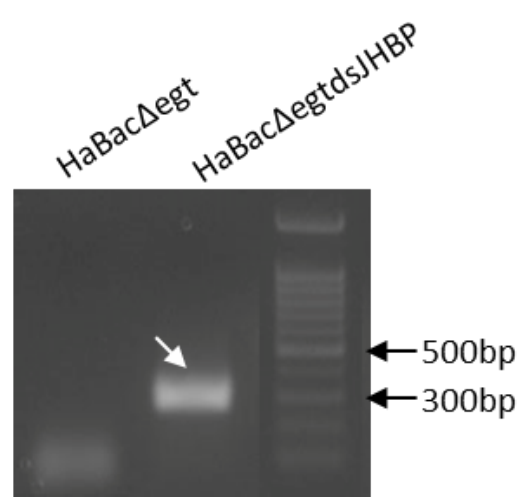

(b)

Figure 3. Transcription verification of dsRNA fragments in infected cells by reverse transcription (RT-PCR). (a) Detection of dsJHAMT total transcription in transfected insect cells; (b) Detection of dsJHBP transcription in transfected insect cells. HaBac $\Delta$ egt-transfected insect cells were used as control. Total RNA of infected cells was extracted at 6 days after transfection. RT-PCR was performed and PCR products underwent electrophoresis in $1.5 \%$ agarose gel. Arrows point to target bands.

\subsection{Efficacy of Recombinant Helicoverpa Armigera Nucleopolyhedroviruses in Killing H. armigera Larvae}

To analyze the biological activity of recombinant HearNPV, we cultivated budded viruses (BVs) in HzAM1 cells and purified sufficient viral occlusion bodies from H. armigera larvae injected with BVs. Third instar $H$. armigera larvae were infected orally with selected doses of OBs and monitored for mortality. The $50 \%$ lethal concentration $\left(\mathrm{LC}_{50}\right)$ value of vHaBac $\Delta$ egtdsJHAMT was 
$1.24 \times 10^{4} \mathrm{OBs} / \mathrm{mL}$, which was just $15.3 \%$ of the control virus vHaBac $\Delta$ egt value of $8.12 \times 10^{4} \mathrm{OBs} / \mathrm{mL}$ (Table 1). Similarly, the $\mathrm{LC}_{50}$ value of vHaBac $\Delta$ egtdsJHBP $\left(2.26 \times 10^{4} \mathrm{OBs} / \mathrm{mL}\right)$ was only $27.8 \%$ of the control value (Table 1). Statistical analysis indicated that the difference in the $\mathrm{tLC}_{50}$ value between $\mathrm{vHaBac} \Delta$ egtdsJHAMT and $\mathrm{vHaBac} \Delta$ egt was significant $(z=4.15, p<0.05)$, as was the difference between vHaBac $\Delta$ egtdsJHBP and vHaBac $\Delta$ egt $(z=7.27, p<0.05)$.

Table 1. The 50\% lethal concentration $\left(\mathrm{LC}_{50}\right)$ values for recombinant Helicoverpa armigera nucleopolyhedroviruses (HearNPVs) in third instar Helicoverpa armigera.

\begin{tabular}{|c|c|c|c|c|}
\hline \multirow{2}{*}{ Virus } & \multirow{2}{*}{$\mathrm{LC}_{50}\left(\times 10^{4} \mathrm{OBs} / \mathrm{mL}\right)$} & \multirow{2}{*}{$\mathrm{SE}$} & \multicolumn{2}{|c|}{ 95\% Confidence Limit } \\
\hline & & & Upper Bound & Lower Bound \\
\hline vHaBac $\Delta$ egtdsJHAMT & 1.24 & 0.49 & 2.42 & 0.49 \\
\hline vHaBac $\Delta$ egtdsJHBP & 2.26 & 0.81 & 4.21 & 1.05 \\
\hline vHaBac $\Delta$ egt & 8.12 & 1.16 & 10.7 & 6.16 \\
\hline
\end{tabular}

$\mathrm{LC}_{50}$ : 50\% lethal concentration; OBs: occlusion bodies; HearNPV: Helicoverpa armigera nucleopolyhedrovirus; SE: standard error.

Then we performed a survival analysis on the third instar H. armigera larvae that were fed HearNPVs. The virus concentration used in this experiment was 100 times the $\mathrm{LC}_{50}$, and the number of dead insects was recorded until all larvae died or pupated. The result showed that the survival time of larvae infected with vHaBac $\Delta$ egtdsJHBP was greatly reduced (Figure 4), and the $\mathrm{LT}_{50}$ value of vHaBac $\Delta$ egtdsJHBP $(65 \mathrm{~h})$ had a significant $\left(\chi^{2}=111.7, p<0.01\right)$ decrease of $45.8 \%$ compared to vHaBac $\Delta$ egt (Table 2), while the survival time of larvae infected with vHaBac $\Delta$ egtdsJHAMT was similar to that of vHaBac $\Delta$ egt, as well as the $\mathrm{LT}_{50}$ values of vHaBac $\Delta$ egtdsJHAMT $(120 \mathrm{~h})$ and vHaBac $\Delta$ egt (120 h).

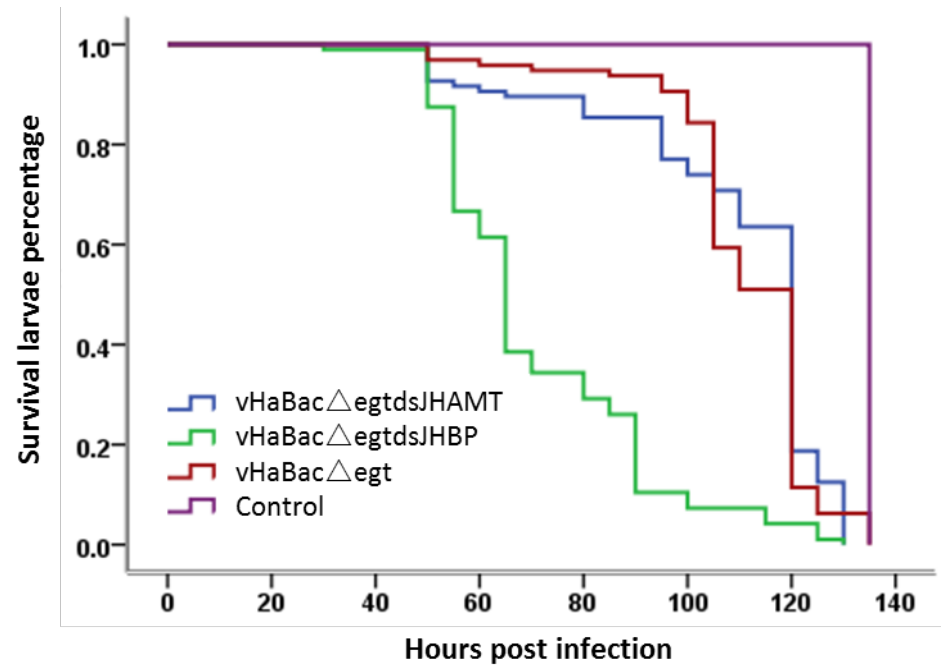

Figure 4. Survival analysis of third instar H. armigera larvae feeding with OBs of baculoviruses. The concentration of OBs was 100 times the $\mathrm{LC}_{50}$ value. The observation continued until all larvae died or pupated. Larvae feeding without baculovirus were used as controls; a total of 96 larvae were tested for each virus. Survival analysis were performed using the Kaplan-Meier estimator by SPSS 22.0. 
Table 2. $\mathrm{LT}_{50}$ values for recombinant HearNPVs in third instar Helicoverpa armigera.

\begin{tabular}{|c|c|c|c|c|}
\hline \multirow{2}{*}{ Virus } & \multirow{2}{*}{$\mathrm{LT}_{50}$ (Hours Post Infection) } & \multirow{2}{*}{ SE } & \multicolumn{2}{|c|}{ 95\% Confidence Limit } \\
\hline & & & Upper Bound & Lower Bound \\
\hline vHaBac $\Delta$ egtdsJHAMT & 120 & 0.89 & 118.26 & 121.74 \\
\hline vHaBac $\Delta$ egtdsJHBP & 65 & 1.08 & 62.88 & 67.12 \\
\hline $\mathrm{vHaBac} \Delta$ egt & 120 & 1.02 & 118.01 & 122.00 \\
\hline
\end{tabular}

Median lethal time value was calculated using the Kaplan-Meier estimator and further compared using a log-rank test (Mantel-Cox) by SPSS 22.0. SE: standard error.

\subsection{Downregulation of RNA Interference Target Gene Expression in Infected H. armigera Larvae}

We used quantitative real-time PCR (qRT-PCR) to detect the transcription level of RNAi target genes in $H$. armigera larvae that were infected by feeding with OBs for two days. The results showed that the mRNA level of HaJHAMT was suppressed in larvae infected with vHaBac $\Delta$ egtdsJHAMT, in contrast to the control virus vHaBac $\Delta$ egt (Figure 5a). Similarly, we found decreased expression of $H a J H B P$ in larvae infected with vHaBac $\Delta$ egtdsJHBP (Figure $5 b$ ).

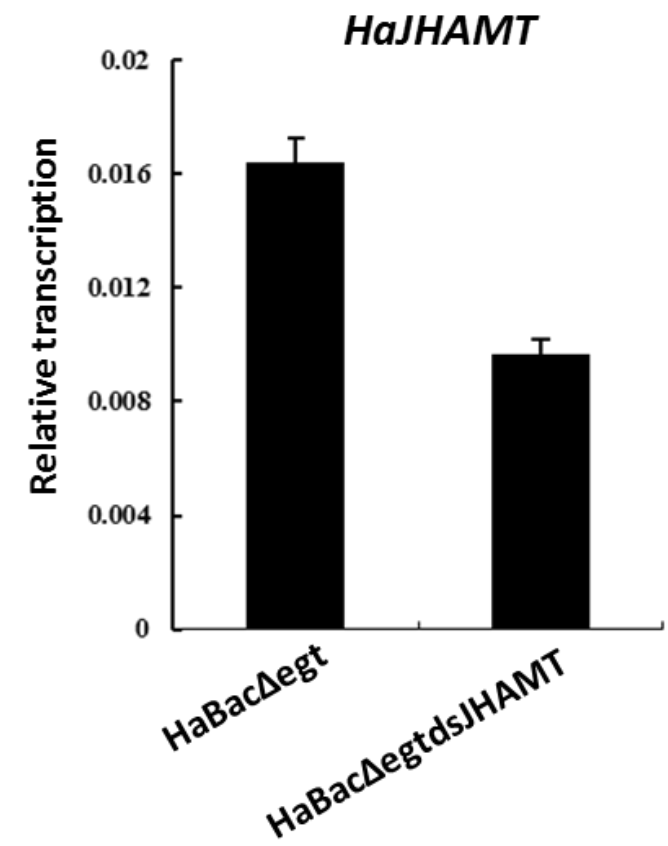

(a)

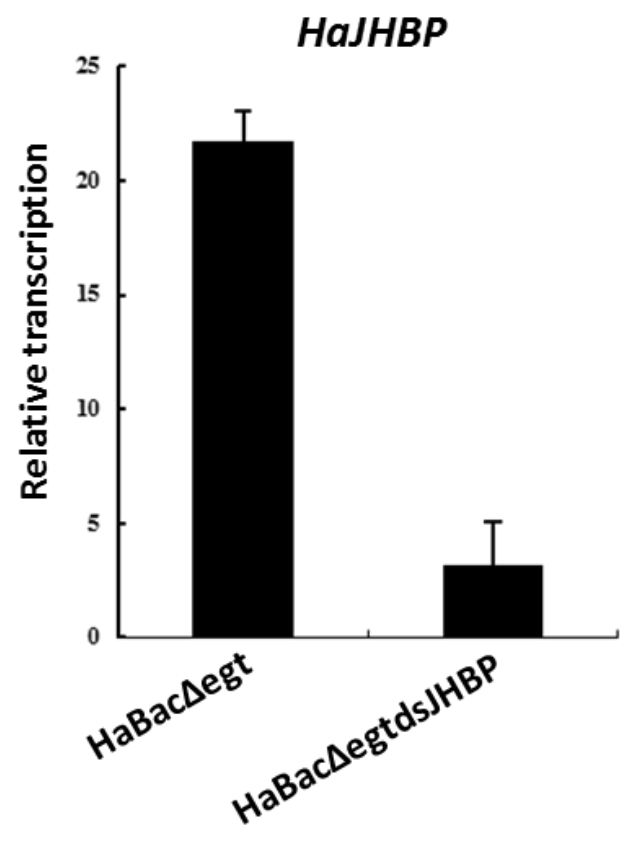

(b)

Figure 5. qRT-PCR analysis of RNA interference (RNAi) target genes in Helicoverpa armigera infected with vHaBac $\triangle$ egtdsJHAMT, vHaBac $\triangle$ egtdsJHBP, and vHaBac $\triangle$ egt. (a) Detection of HaJHAMT; (b) Detection of $H a J H B P$. The cDNA from third instar larval at $48 \mathrm{~h}$ post infection were analyzed. ACTIN was selected as the reference gene. Data represent the ratio of gene expression to ACTIN. Bars show means and standard errors (three independent replicates).

\subsection{Effect on Hormone-Related Downstream Genes in Infected H. armigera Larvae}

To investigate the effect of recombinant HearNPVs on other hormone-related gene larvae, we detected mRNA levels of several key genes in orally infected $H$. armigera larvae using qRT-PCR, including the $\mathrm{JH}$ receptor gene HaMet, ultraspiracle (HaUSP), a potential candidate for the $\mathrm{JH}$ receptor, JH regulated gene RNA-binding protein $(H a R B P)$, and ecdysone receptor gene $H a E c R$. We found that transcription of HaRBP and HaUSP increased in vHaBac $\Delta$ egtdsJHBP-infected larvae compared to the control (vHaBac $\Delta$ egt-infected larvae), while HaUSP expression was reduced in 
vHaBac $\Delta$ egtdsJHAMT-infected larvae (Figure 6). Additionally, HaEcR expression decreased in both recombinant HearNPV-infected larvae.

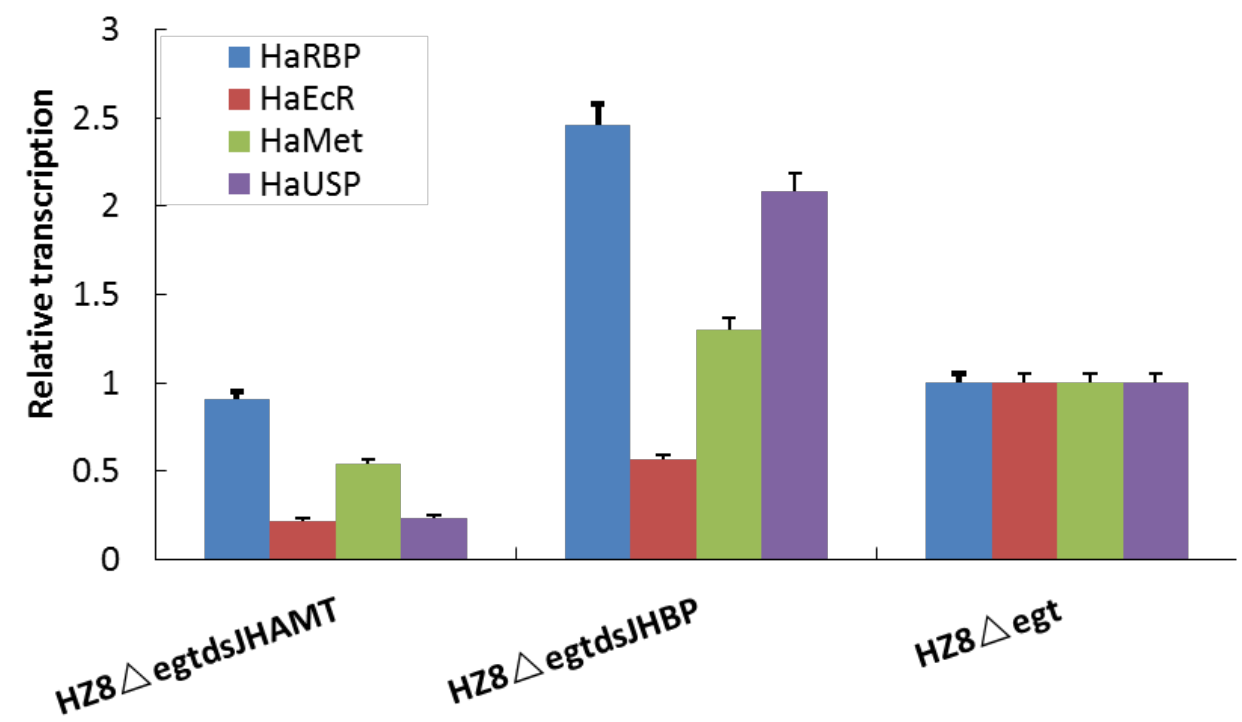

Figure 6. The qRT-PCR detection of genes involved in hormone pathways in third instar Helicoverpa armigera larvae. After $48 \mathrm{~h}$ of feeding with vHaBac $\Delta$ egtdsJHAMT, vHaBac $\Delta$ egtdsJHBP, and vHaBac $\Delta$ egt, gene expression was analyzed by qRT-PCR. ACTIN was used as an internal standard to normalize the level of gene expression. Data of the histogram represents the ratio of gene expression to that in the control (vHaBac $\Delta$ egt infected larvae). Bars show means and standard errors (three independent replicates).

\section{Discussion}

Baculoviruses are the most widely used virus insecticide in the world. However, in contrast to plant- or bacteria-mediated RNAi, the application of baculovirus as a dsRNA delivery vehicle for pest control has attracted far less attention [26]. In fact, it was verified 10 years ago that recombinant baculovirus expressing dsGFP can trigger RNAi and silence green fluorescence protein (GFP) in insect culture cells [29]. In this study, we successfully improved the insecticidal activity of baculovirus by expressing dsJHAMT and dsJHBP in it (Figure 1). Previous research indicated that baculovirus-mediated RNAi was not suitable for functionally characterizing genes of the lepidopteran insects, since the infection effects might mask the potentially produced phenotypes associated with RNAi [30]. Obviously, this phenomenon has no effect when baculovirus-mediated RNAi is applied to control pests.

The combined actions of $\mathrm{JH}$ and ecdysteroids are crucial in regulating insect molting and metamorphosis. The presence of JH during larval molting prevents pupation [31]. JHAMT is an irreplaceable enzyme for the unique reaction steps in juvenile hormone synthesis [32,33]. On the other hand, nearly every JH molecule binds to JHBP in hemolymph when transported from corpora allata cells, the JH synthesizing place, to its sites of action [34,35]. JHBP also protects the chemically labile JH from nonspecific enzymatic degradation [36]. Suppressing the expression of the two genes, HaJHAMT and $H a J H B P$, disturbs JH concentration in cotton bollworm and further affects larval survival [24]. Therefore, we chose to inhibit the expression of these two genes via baculovirus-mediated RNAi to improve the insecticidal activity of baculovirus.

In this study, we constructed two recombinant bacmids containing a dsJHAMT and dsJHBP expression cassette. To ensure that RNAi occurred in cotton bollworm as soon as possible after infection and persisted throughout the infection, dsRNA expression was driven by two baculovirus promoters, the early op 166 promoter and the late $p 10$ promoter (Figure 1). After HzAM1 cells were transfected with these recombinant bacmids, green fluorescence and OBs were observed in infected 
cells (Figure 2). These results indicate that the insertion of dsRNA did not affect virus infection and OB formation in HzAM1 cells. Further examination of infected cells by RT-PCR (Figure 3) confirmed the transcription of dsRNA as expected.

Bioassay results confirmed that our strategy to modify baculovirus was successful. Both recombinant HearNPVs in this study exhibited a large reduction in $\mathrm{LC}_{50}$ value compared to the control (Table 1 ), which shows that dsRNA expressed by baculoviruses played an extra insecticidal role in comparison with the control virus. However, for the LT50 bioassay, the result of vHaBac $\Delta$ egtdsJHBP was significantly reduced, while there was no significant difference between vHaBac $\Delta$ egtdsJHAMT and the control (Table 2). We suspected that this might be due to the lower virus concentrations used for infection than that of the control. The specific virus concentrations (100 times the $\mathrm{LC}_{50}$ ) were $2.26 \times 10^{6} \mathrm{OBs} / \mathrm{mL}$ for vHaBac $\Delta$ egtdsJHBP, $1.24 \times 10^{6} \mathrm{OBs} / \mathrm{mL}$ for vHaBac $\Delta$ egtdsJHAMT, and $8.12 \times 10^{6} \mathrm{OBs} / \mathrm{mL}$ for the control, respectively. Further testing indicated that the transcription of dsRNA target genes HaJHAMT and HaJHBP in H. armigera larvae was suppressed in cotton bollworm larvae fed with recombinant OBs (Figure 5). This might reduce JH concentration in H. armigera [24]. Considering there was cross-talk between JH and ecdysteroid signaling [31], and that the transcription of other genes (HaECR et al.) involved in insect hormone signaling pathway was indeed altered at $48 \mathrm{~h}$ post-infection with recombinant HearNPVs (Figure 6), ecdysteroid function might also be affected in larvae. Therefore, we proposed a hypothesis. Recombinant HearNPVs could effectively express dsJHAMT and dsJHBP (Figure 3), and reduce target genes mRNA levels in infected larvae (Figure 5). This may lead to a decrease in JH concentration [24] and interfere with the hormonal equilibrium in infected host, which in turn might affect the growth and development of the larvae and ultimately results in larvae death. Obviously, this additional insecticidal effect will increase the insecticidal activity of recombinant HearNPVs. Specific to this study, it was reflected that recombinant HearNPVs had lower $\mathrm{LC}_{50}$ values and a shorter $\mathrm{LT}_{50}$ value than the control vHaBac $\Delta$ egt (Tables 1 and 2). In agreement with our results, recombinant baculoviruses expressing juvenile hormone esterase (JHE) or a mutant JHE (more stable in hemolymph than wild-type JHE) showed a dramatic reduction in $\mathrm{LC}_{50}$ in comparison to larvae infected with wild-type AcMNPV [16,37]. JHE is a catalytically inactive insect enzyme, and its overexpression decreases the concentration of $\mathrm{JH}$ [38]. Besides, the expression of dsJHBP rather than dsJHAMT could speed up the insecticidal activity of baculovirus in our research (Figure 4 and Table 2), possibly because JHBP is located farther downstream of the JH signaling pathway and has a faster impact on the JH titer than JHAMT.

In the past, three major approaches were pursued to improve the speed of baculovirus killing action by genetic engineering technology: (1) introduction of a gene encoding an insect-specific toxin, hormone, or enzyme into the baculovirus genome; (2) deletion of baculovirus genes that affect infection; and (3) incorporation of a toxin, like Bt toxin, into the polyhedrin matrix $[2,39,40]$. In this paper, we provide a new strategy: disturbing insect hormones by baculovirus-mediated RNAi. This strategy can be combined with previous improvements. For example, the baculovirus used in this study has already had the ecdysteroid UDP-glucosyltransferase (EGT) gene deleted, which may result in a reduction in food consumption and time to kill the host compared with wild-type HearNPV [41,42]. Laboratory analysis in this work has demonstrated that the recombinant HearNPV expressing dsJHBP could further shorten the killing time of $H$. armigera larvae with fewer occlusion bodies comparison to $\mathrm{vHaBac} \Delta$ egt. These characteristics make it possible for $\mathrm{vHaBac} \Delta$ egtdsJHBP to be further developed into a new bioinsecticide. However, we need to consider the production of baculoviruses for industrial application. The number of OB produced by each larva may be reduced, since larvae will die earlier after infection with $v H a B a c \Delta$ egtdsJHBP. Perhaps it would be more advantageous for industrialization to replace $\mathrm{vHaBac} \Delta$ egt with wildtype baculoviruses, which harbor the egt that prevents molting and prolongs the larval stage of infected insects, and allows for production of more progeny virus. Besides, as the recombinant baculovirus have stronger insecticidal activity, its application amount will also decrease. 


\section{Materials and Methods}

\subsection{Insects, Cell Line, and Viruses}

Helicoverpa armigera larvae supplied by the Experimental Animal Center of Wuhan Institute of Virology, Chinese Academy of Sciences, were reared on an artificial diet at $26{ }^{\circ} \mathrm{C}, 60 \%$ humidity, and a $16 \mathrm{~h} / 8 \mathrm{~h}$ photoperiod. Helioverpa zea cell line HzAM1 cells [43], kindly provided by Dr. Hu Zhihong (Wuhan Institute of Virology, Wuhan, China), were maintained in Grace's insect medium supplemented with $10 \%$ FBS (fetal calf serum) at $28^{\circ} \mathrm{C}$. The infectious bacmid of HearNPV, HaBacHz8- $\Delta$ egt (the $e g t$ locus was replaced by eGFP gene) [42], and intermediate plasmids pFBD-HaPH and pFB-op166 used in this study were also presented by Dr. Hu Zhihong [44,45].

\subsection{Construction of Donor Plasmids}

The baculovirus early promoter op166 was amplified by PCR from plasmid pFB-op166 using primers OPF and OPR (see Supplementary Table S1). The obtained $184 \mathrm{bp}$ amplicon was cloned into the pMD-18T vector for sequence verification. After digesting with NcoI and SmaI, op166 promoter was inserted into the vector pFBD-HaPH downstream of the $p 10$ promoter to obtain the donor plasmid precursor pFBD-HaPH-op166. Hairpin RNAi constructs dsJHAMT and dsJHBP, as previously described [24], were excised by Sse8387I (blunting) from plasmids stored in our lab, and then cloned into the PvuII enzyme site of pFBD-HaPH-op166 downstream of the op166 promoter to produce donor plasmids pFBD-HaPH-op166-dsJHAMT and pFBD-HaPH-op166-dsJHBP, respectively.

\subsection{Construction of Recombinant Bacmids}

These two donor plasmids were transformed into E. coli DH10B-competent cells harboring a $\mathrm{HaBacHz} 8-\Delta$ egt bacmid and helper plasmid expressing transposase, respectively. Correspondingly, the resulting recombinant bacmids produced by transposition were named HaBac $\Delta$ egt-dsJHAMT and $\mathrm{HaBac} \Delta$ egtdsJHBP. According to the Bac-to-Bac ${ }^{\mathrm{TM}}$ system manual, the colonies were selected in the presence of kanamycin, gentamicin, tetracycline, and X-Gal. White recombinants were picked and then checked by PCR using M13 forward primer and gene-specific primers.

\subsection{Generation of Budded Viruses}

Bacmid DNAs of HaBac $\Delta$ egt-dsJHAMT, HaBac $\Delta$ egtdsJHBP, and HaBac $\Delta$ egt were purified from $5 \mathrm{~mL}$ cultures. A total of $5 \mu \mathrm{g}$ bacmid DNA was used to transfect $5 \times 10^{5} \mathrm{HzAM} 1$ cells with the aid of $10 \mu \mathrm{L}$ of lipofectin, following the manufacturer's instructions (Invitrogen, Carlsbad, CA, USA). When cultured at $27{ }^{\circ} \mathrm{C}$ for three days, transfected cells were viewed for fluorescence under an inverted fluorescence microscope (Nikon TE-300, Tokyo, Japan). After six days' culture, the cells were centrifuged at $4000 \mathrm{rpm}$ for $10 \mathrm{~min}$. The supernatant was collected as the first-generation virus and used to infect HzAM1 cells at a rate of 0.1 multiplicity of infection (MOI). When the yield of budded viruses (BVs) reached the peak $(96 \mathrm{~h})$, the supernatant, as the second-generation virus, was collected, and used to obtain the third-generation virus. The resulting BVs were named vHaBac $\Delta$ egt-dsJHAMT, vHaBac $\Delta$ egtdsJHBP, and vHaBac $\Delta$ egt and tested for $50 \%$ tissue culture infective dose $\left(\right.$ TCID $\left._{50}\right)$ by endpoint dilution assay. All viruses were kept in the dark at $4{ }^{\circ} \mathrm{C}$ for further experiments.

\subsection{Detection of dsRNA Transcripts in Infected Cells}

To make sure the dsRNA expression cassette inserted into the HearNPV genome was active, reverse transcription PCR (RT-PCR) was used to detect dsRNA expression. The total RNA was extracted from infected HzAM1 cells at 6 dpi with Trizol (Invitrogen), according to the user manual. Next, $1.0 \mu \mathrm{g}$ of total RNA was used to synthesize cDNA with a GoScript ${ }^{\mathrm{TM}}$ reverse transcription system (Promega, Madison, WI, USA) and oligo(dT) anchor primer. Primer pairs JTF and Pin and 
Pin and JPR (see Supplementary Table S1) were used to detect the transcription of dsJHAMT and dsJHBP, respectively.

\subsection{Purification of Viral Occlusion Bodies}

Third instar Helicoverpa armigera larvae were infected via microinjection with the third-generation BVs (a titer of $1 \times 10^{7} \mathrm{TCID}_{50} \mathrm{~mL}^{-1}, 5 \mu \mathrm{L}$ for each larva), vHaBac $\Delta$ egtdsJHAMT, vHaBac $\Delta$ egtdsJHBP, and $\mathrm{vHaBac} \Delta$ egt. After about five days, cadavers of the larva that died from infection were collected and mashed. After adding some distilled water, the mixture was centrifuged at $300 \times g$ for $10 \mathrm{~min}$ to collect the supernatant. Then the supernatant was centrifuged again at $3000 \times g$ for $30 \mathrm{~min}$. The precipitate was resuspended in distilled water and thoroughly mixed, which was the purified OBs. The purified OBs were diluted and counted under a microscope using a hemocytometer. Samples were counted three times and averaged.

\subsection{Transcription Analysis of Genes in Infected H. armigera Larvae}

The total RNA was extracted from three larvae that were infected with OBs and reared for two days. First-strand cDNA was synthesized by a GoScript ${ }^{\mathrm{TM}}$ reverse transcription system (Promega) and diluted 10 times as a template. To measure transcription of HaJHAMT, HaJHBP, and related genes in OB-feeding H. armigera larvae, we performed quantitative real-time PCR (qRT-PCR) using a LightCycler 480 system (Roche, Basel, Switzerland) with LightCycler 480 SYBR Green I Master (Roche) following a two-step protocol: $95^{\circ} \mathrm{C}$ for $10 \mathrm{~min}, 45$ cycles of denaturation at $95^{\circ} \mathrm{C}$ for $10 \mathrm{~s}$, annealing at $58^{\circ} \mathrm{C}$ for $20 \mathrm{~s}$, and extension at $72{ }^{\circ} \mathrm{C}$ for $20 \mathrm{~s}$. We used ACTIN as the reference gene, and calculated transcription relative to ACTIN with the $2^{-\Delta \Delta C t}$ method [46]. The primers used for qRT-PCR are listed in Supplementary Table S1.

\subsection{Bioassays}

$\mathrm{LC}_{50}$ values of recombinant HearNPVs were determined by the droplet feeding method [47]. Late second instar $\mathrm{H}$. armigera larvae were placed in 24 -well sterile plates and starved overnight at $28^{\circ} \mathrm{C}$. The following morning, larvae that had molted third instar were used for bioassays. The polyhedrosis virus was set to $3 \times 10^{5} \mathrm{OBs} / \mathrm{mL}, 1 \times 10^{5} \mathrm{OBs} / \mathrm{mL}, 3 \times 10^{4} \mathrm{OBs} / \mathrm{mL}, 1 \times 10^{4} \mathrm{OBs} / \mathrm{mL}$, and $3 \times 10^{3} \mathrm{OBs} / \mathrm{mL}$ suspensions. The starving third instar larvae were fed with the different suspensions of OBs mixed with $4 \%$ sucrose and $1 \mathrm{mg} / \mathrm{mL}$ blue dye (erioglaucine disodium salt, Sigma-Aldrich, St. Louis, MO, USA). The larvae whose midgut changed to blue in $10 \mathrm{~min}$ were transferred individually into a 24-well plate containing fresh artificial diet. The mortality rate was observed twice a day until all larvae died or pupated. Each OB suspension concentration was tested using 24 larvae and the experiment was performed twice. The $\mathrm{LC}_{50}$ values were analyzed by Probit regression analysis and compared by $z$-test in SPSS (IBM SPSS Statistics 22.0, Armonk, NY, USA).

$\mathrm{LT}_{50}$ values of recombinant HearNPV were also tested by a droplet feeding method at a high $\mathrm{OB}$ concentration $\left(100 \times \mathrm{LC}_{50}\right)$. Second instar $H$. armigera larvae were infected as mentioned above. Time 0 was defined as the point where larvae were transferred to the fresh diet. Then we observed and recorded the number of deaths at 07:00, 13:00, 18:00, and 23:00 every day until all the larvae died or pupated. Each HearNPV was tested using 96 larvae. LT $_{50}$ values were calculated using the Kaplan-Meier estimator and further compared by a log-rank test (Mantel-Cox) in SPSS 22.0 [48].

\section{Conclusions}

In conclusion, the results obtained show that recombinant HearNPV expressing dsJHAMT or dsJHBP successfully suppressed target gene expression in infected cotton bollworm, and these recombinant HearNPVs had higher insecticidal activity than the unmodified baculovirus. Especially for vHaBac $\Delta$ egtdsJHBP, the insecticidal speed increased by $45.8 \%$, while the toxicity more than doubled. These data show the possibility for applying engineered baculoviruses in cotton bollworm control, and 
this research provides a new strategy for pest management by interfering with insect hormone-related genes via baculovirus-mediated RNAi.

\section{Patents}

Two patents resulted from the work reported in this paper. The patent authorization numbers are ZL201210576020.4 and ZL201210576007.9.

Supplementary Materials: Supplementary materials can be found at http:/ / www.mdpi.com/1422-0067/20/2/ $419 / \mathrm{s} 1$.

Author Contributions: Conceptualization, Z.Z.; investigation, Z.L. and X.W. (Xiaofang Wang); writing—original draft preparation, Z.L. and M.N.; writing-review and editing, Z.Z., L.Z., and Y.D.; supervision, Z.Z. and L.Z.; project administration, X.W. (Xiaoli Wei); funding acquisition, Z.Z. and Y.D.

Funding: This research was funded by the Ministry of Agriculture and Rural Affairs of China (grant numbers 2016ZX08001-001 and 2016ZX08010-002) and the State Key Laboratory of Plant Genomics of China (grant number SKLPG2016B-21).

Acknowledgments: We thank Zhihong Hu and Xiulian Sun for providing materials and technical support used for experiments.

Conflicts of Interest: The authors declare no conflict of interest. The funders had no role in the design of the study; in the collection, analysis, or interpretation of data; in the writing of the manuscript, or in the decision to publish the results.

\section{Abbreviations}

$\begin{array}{ll}\text { bacmid } & \text { baculovirus plasmid } \\ \mathrm{Bt} & \text { Bacillus thuringiensis } \\ e G F P & \text { enhanced green fluorescence protein gene } \\ G m^{R} & \text { gentamicin resistance gene } \\ \mathrm{Cm}^{R} & \text { chloramphenicol resistance gene } \\ \mathrm{TCID}_{50} & 50 \% \text { tissue culture infective dose } \\ \mathrm{PIB} & \text { polyhedral inclusion body } \\ \mathrm{UDP} & \text { uridine diphosphate }\end{array}$

\section{References}

1. Oerke, E.C. Crop losses to pests. J. Agric. Sci. 2006, 144, 31-43. [CrossRef]

2. Beascatena, A.; Sánchezmirón, A.; Garcíacamacho, F.; Contrerasgómez, A.; Molinagrima, E. Baculovirus biopesticides: An overview. J. Anim. Plant Sci. 2014, 24, 362-373.

3. Douglas, A.E. Strategies for enhanced crop resistance to insect pests. Annu. Rev. Plant Biol. 2018, 69, 637-660. [CrossRef]

4. Armenta, R.; Martínez, A.M.; Chapman, J.W.; Magallanes, R.; Goulson, D.; Caballero, P.; Cave, R.D.; Cisneros, J.; Valle, J.; Castillejos, V.; et al. Impact of a nucleopolyhedrovirus bioinsecticide and selected synthetic insecticides on the abundance of insect natural enemies on maize in southern Mexico. J. Econ. Entomol. 2003, 96, 649-661. [CrossRef] [PubMed]

5. Makkonen, K.E.; Airenne, K.; Yla-Herttulala, S. Baculovirus-mediated gene delivery and RNAi applications. Viruses 2015, 7, 2099-2125. [CrossRef] [PubMed]

6. Fuller, E.; Elderd, B.D.; Dwyer, G. Pathogen Persistence in the Environment and Insect-Baculovirus Interactions: Disease-Density Thresholds, Epidemic Burnout, and Insect Outbreaks. Am. Nat. 2012, 179, E70-E96. [CrossRef] [PubMed]

7. Haase, S.; Sciocco-Cap, A.; Romanowski, V. Baculovirus insecticides in Latin America: Historical overview, current status and future perspectives. Viruses 2015, 7, 2230-2267. [CrossRef]

8. Szewczyk, B.; Hoyos-Carvajal, L.; Paluszek, M.; Skrzecz, I.; Souza, M.L.D. Baculoviruses-Re-emerging biopesticides. Biotechnol. Adv. 2006, 24, 143-160. [CrossRef]

9. Moscardi, F. Assessment of the application of baculoviruses for control of Lepidoptera. Annu. Rev. Entomol. 1999, 44, 257-289. [CrossRef] 
10. Stewart, L.M.D.; Hirst, M.; Ferber, M.L.P.; Merryweather, A.T.; Cayley, P.J.; Possee, R.D. Construction of an improved baculovirus insecticide containing an insect-specific toxin gene. Nature 1991, 352, 85-88. [CrossRef]

11. Tomalski, M.D.; Miller, L.K. Insect paralysis by baculovirus-mediated expression of a mite neurotoxin gene. Nature 1991, 352, 82-85. [CrossRef] [PubMed]

12. Paul Pener, M. Insect Growth Regulators. In Encyclopedia of Pest Management; CRC Press: Boca Raton, FL, USA, 2002. [CrossRef]

13. Maeda, S. Increased insecticidal effect by a recombinant baculovirus carrying a synthetic diuretic hormone gene. Biochem. Biophys. Res. Commun. 1989, 165, 1177-1183. [CrossRef]

14. Eldridge, R.; Horodyski, F.M.; Morton, D.B.; O’Reilly, D.R.; Truman, J.W.; Riddiford, L.M.; Miller, L.K. Expression of an eclosion hormone gene in insect cells using baculovirus vectors. Insect Biochem. 1991, 21, 341-351. [CrossRef]

15. O’Reilly, D.R.; Kelly, T.J.; Masler, E.P.; Thyagaraja, B.S.; Moy Robson, R.; Shaw, T.C.; Miller, L.K. Overexpression of Bombyx mori prothoracicotropic hormone using baculovirus vectors. Insect Biochem. Mol. Biol. 1995, 25, 475-485. [CrossRef]

16. Hammock, B.D.; Bonning, B.C.; Possee, R.D.; Hanzlik, T.N.; Maeda, S. Expression and effects of the juvenile hormone esterase in a baculovirus vector. Nature 1990, 344, 458-461. [CrossRef]

17. O'Reilly, D.R.; Miller, L.K. Improvement of a baculovirus pesticide by deletion of the EGT gene. Nat. Biotechnol. 1991, 9, 1086-1089. [CrossRef]

18. O'Reilly, D.R. Baculovirus-encoded ecdysteroid UDP-glucosyltransferases. Insect Biochem. Mol. Biol. 1995, 25, 541-550. [CrossRef]

19. Mello, C.C.; Conte, D. Revealing the world of RNA interference. Nature 2004, 431, 338-342. [CrossRef]

20. Baum, J.A.; Bogaert, T.; Clinton, W.; Heck, G.R.; Feldmann, P.; Ilagan, O.; Johnson, S.; Plaetinck, G.; Munyikwa, T.; Pleau, M.; et al. Control of coleopteran insect pests through RNA interference. Nat. Biotechnol. 2007, 25, 1322-1326. [CrossRef]

21. Mao, Y.B.; Cai, W.J.; Wang, J.W.; Hong, G.J.; Tao, X.Y.; Wang, L.J.; Huang, Y.P.; Chen, X.Y. Silencing a cotton bollworm P450 monooxygenase gene by plant-mediated RNAi impairs larval tolerance of gossypol. Nat. Biotechnol. 2007, 25, 1307-1313. [CrossRef]

22. Kolliopoulou, A.; Swevers, L. Recent progress in RNAi research in Lepidoptera: Intracellular machinery, antiviral immune response and prospects for insect pest control. Curr. Opin. Insect Sci. 2014, 6, 28-34. [CrossRef]

23. Joga, M.R.; Zotti, M.J.; Smagghe, G.; Christiaens, O. RNAi efficiency, systemic properties, and novel delivery methods for pest insect control: What we know so far. Front. Physiol. 2016, 7, 553. [CrossRef] [PubMed]

24. Ni, M.; Ma, W.; Wang, X.; Gao, M.; Dai, Y.; Wei, X.; Zhang, L.; Peng, Y.; Chen, S.; Ding, L.; et al. Next-generation transgenic cotton: Pyramiding RNAi and Bt counters insect resistance. Plant Biotechnol. J. 2017, 15, 1204-1213. [CrossRef] [PubMed]

25. Hitchon, A. Environmental safety assessment considerations for plants expressing novel insecticidal RNAi traits in Canada. In Proceedings of the 2016 International Congress of Entomology, Orlando, FL, USA, 25-30 September 2016.

26. Kolliopoulou, A.; Taning, C.N.T.; Smagghe, G.; Swevers, L. Viral delivery of dsRNA for control of insect agricultural pests and vectors of human disease: Prospects and challenges. Front. Physiol. 2017, 8, 399. [CrossRef] [PubMed]

27. Tay, W.T.; Soria, M.F.; Walsh, T.; Thomazoni, D.; Silvie, P.; Behere, G.T.; Anderson, C.; Downes, S. A brave new world for an old world pest: Helicoverpa armigera (Lepidoptera: Noctuidae) in Brazil. PLoS ONE 2013, 8, e80134. [CrossRef] [PubMed]

28. Popham, H.J.R.; Nusawardani, T.; Bonning, B.C. Introduction to the Use of Baculoviruses as Biological Insecticides, 3rd ed.; Murhammer, D.W., Ed.; Springer: New York, NY, USA, 2016; pp. 383-392. [CrossRef]

29. Huang, Y.; Deng, F.; Hu, Z.; Vlak, J.; Wang, H. Baculovirus-mediated gene silencing in insect cells using intracellularly produced long double-stranded RNA. J. Biotechnol. 2007, 128, 226-236. [CrossRef] [PubMed]

30. Kontogiannatos, D.; Swevers, L.; Maenaka, K.; Park, E.Y.; Iatrou, K.; Kourti, A. Functional characterization of a juvenile hormone esterase related gene in the moth Sesamia nonagrioides through RNA interference. PLoS ONE 2013, 8, e73834. [CrossRef]

31. Jindra, M.; Palli, S.R.; Riddiford, L.M. The juvenile hormone signaling pathway in insect development. Annu. Rev. Entomol. 2013, 58, 181-204. [CrossRef] 
32. Bellés, X.; Martín, D.; Piulachs, M.D. The mevalonate pathway and the synthesis of juvenile hormone in insects. Annu. Rev. Entomol. 2005, 50, 181-199. [CrossRef]

33. Minakuchi, C.; Namiki, T.; Yoshiyama, M.; Shinoda, T. RNAi-mediated knockdown of juvenile hormone acid O-methyltransferase gene causes precocious metamorphosis in the red flour beetle Tribolium castaneum. FEBS J. 2008, 275, 2919-2931. [CrossRef]

34. Trowell, S.C. High affinity juvenile hormone carrier proteins in the haemolymph of insects. Comp. Biochem. Physiol. Part B Comp. Biochem. 1992, 103, 795-807. [CrossRef]

35. Hidayat, P.; Goodman, W.G. Juvenile hormone and hemolymph juvenile hormone binding protein titers and their interaction in the hemolymph of fourth stadium Manduca sexta. Insect Biochem. Mol. Biol. 1994, 24, 709-715. [CrossRef]

36. Suzuki, R.; Fujimoto, Z.; Shiotsuki, T.; Tsuchiya, W.; Momma, M.; Tase, A.; Miyazawa, M.; Yamazaki, T. Structural mechanism of JH delivery in hemolymph by JHBP of silkworm, Bombyx mori. Sci. Rep. 2011, 1, 133. [CrossRef] [PubMed]

37. El-Sheikh, E.-S.A.; Kamita, S.G.; Vu, K.; Hammock, B.D. Improved insecticidal efficacy of a recombinant baculovirus expressing mutated JH esterase from Manduca sexta. Biol. Control 2011, 58, 354-361. [CrossRef]

38. Kamita, S.G.; Hammock, B.D. Juvenile hormone esterase: Biochemistry and structure. J. Pest. Sci. 2010, 35, 265-274. [CrossRef] [PubMed]

39. Inceoglu, A.B.; Kamita, S.G.; Hammock, B.D. Genetically modified baculoviruses: A historical overview and future outlook. Adv. Virus Res. 2006, 68, 323-360. [CrossRef]

40. Chang, J.H.; Choi, J.Y.; Jin, B.R.; Roh, J.Y.; Olszewski, J.A.; Seo, S.J.; O’Reilly, D.R.; Je, Y.H. An improved baculovirus insecticide producing occlusion bodies that contain Bacillus thuringiensis insect toxin. J. Invertebr. Pathol. 2003, 84, 30-37. [CrossRef]

41. Georgievska, L.; Joosten, N.; Hoover, K.; Cory, J.S.; Vlak, J.M.; van der Werf, W. Effects of single and mixed infections with wild type and genetically modified Helicoverpa armigera nucleopolyhedrovirus on movement behaviour of cotton bollworm larvae. Entomol. Exp. Appl. 2010, 135, 56-67. [CrossRef]

42. Si, Y.-H.; Fang, M.-G.; Huang, Y.; Zheng, F.-L.; Li, T.; Hu, Z.-H.; Wang, H.-Z. Construction and characterization of a Helicoverpa armigera nucleopolyhedrovirus bacterial artificial chromosome with deletion of ecdysteroid UDP-glucosyltransferase gene. Biosci. Biotechnol. Biochem. 2007, 71, 2435-2441. [CrossRef]

43. McIntosh, A.H.; Ignoffo, C.M. Characterization of five cell lines established from species of Heliothis. Appl. Entomol. Zool. 1983, 18, 262-269. [CrossRef]

44. Wang, H.; Deng, F.; Pijlman, G.P.; Chen, X.; Sun, X.; Vlak, J.M.; Hu, Z. Cloning of biologically active genomes from a Helicoverpa armigera single-nucleocapsid nucleopolyhedrovirus isolate by using a bacterial artificial chromosome. Virus Res. 2003, 97, 57-63. [CrossRef] [PubMed]

45. Wang, M.; Tan, Y.; Yin, F.; Deng, F.; Vlak, J.M.; Hu, Z.; Wang, H. The F protein of Helicoverpa armigera single nucleopolyhedrovirus can be substituted functionally with its homologue from Spodoptera exigua multiple nucleopolyhedrovirus. J. Gen. Virol. 2008, 89, 791-798. [CrossRef] [PubMed]

46. Livak, K.J.; Schmittgen, T.D. Analysis of relative gene expression data using real-time quantitative PCR and the $2^{-\Delta \Delta C t}$ method. Methods 2001, 25, 402-408. [CrossRef]

47. Hughes, P.R.; van Beek, N.A.M.; Wood, H.A. A modified droplet feeding method for rapid assay of Bacillus thuringiensis and baculoviruses in noctuid larvae. J. Invertebr. Pathol. 1986, 48, 187-192. [CrossRef]

48. Luo, S.; Zhang, Y.; Xu, X.; Westenberg, M.; Vlak, J.M.; Wang, H.; Hu, Z.; Deng, F. Helicoverpa armigera nucleopolyhedrovirus occlusion-derived virus-associated protein, HA100, affects oral infectivity in vivo but not virus replication in vitro. J. Gen. Virol. 2011, 92, 1324-1331. [CrossRef] [PubMed]

(C) 2019 by the authors. Licensee MDPI, Basel, Switzerland. This article is an open access article distributed under the terms and conditions of the Creative Commons Attribution (CC BY) license (http://creativecommons.org/licenses/by/4.0/). 